\title{
My Personal User Interface: A Semantic User-Centric Approach to Manage and Share User Information
}

\author{
Till Plumbaum ${ }^{1}$, Katja Schulz $^{2}$, Martin Kurze ${ }^{2}$, and Sahin Albayrak ${ }^{1}$ \\ ${ }^{1}$ Technische Universität Berlin, DAI-Labor, Berlin, Germany \\ ${ }^{2}$ Deutsche Telekom Laboratories, Berlin, Germany \\ \{Till.Plumbaum, Sahin.Albayrak\} @dai-labor.de, \\ \{Katja.Schulz02, Martin.Kurze\} @telekom.de
}

\begin{abstract}
With the growing impact of the Web 2.0 on our every day life, people start to use more and more different web based services like Facebook or Twitter. Thereby, they generate and distribute personal and social information such as interests, preferences and goals that are stored in a user profile. This leads to open challenges regarding the users ability to keep track of their personal information but it also offers chances to use this data to enhance personalization and recommendations of existing services. This paper presents a usercentric, thus privacy preserving, system to cope with these challenges and a personal user interface (UI) that allows users to manage and share personal data.
\end{abstract}

Keywords: information visualization, recommender systems, data mining, user modeling, user-centered design, ontological engineering, semantic mapping.

\section{Introduction}

With the growing impact of the Web 2.0 on our every day life, people start to use more and more different web based services like Facebook ${ }^{1}$, Twitter ${ }^{2}$, Flickr $^{3}$ or blogs. They use those services to express their opinion, communicate with others and share pictures with friends. Thereby, they generate and distribute personal and social information like interests, social contacts, preferences and personal goals [1]. This user information is usually stored in a user profile deeply buried within every service, only accessible through the User Interface (UI) or API. This affects the users ability to keep track of their personal information. They loose overview what information is stored where and what is public and what private, which leads to open privacy and security challenges. Users who have no overview of the data stored cannot control what data is publicly available and thus, information can be shared by accident. However, the personal information distributed over different services represents an untapped store of knowledge that could be used to enhance personalization and recommendation for existing services. In this work, we present a user-centric, thus privacy preserving, system that

\footnotetext{
${ }^{1} \mathrm{http}: / / \mathrm{www}$. facebook.com

${ }^{2} \mathrm{http}: / /$ twitter.com

${ }^{3}$ http://www.flickr.com/
} 
consists of a semantic layer to aggregate user profiles and a personal user interface to visualize the profile information. The semantic layer aggregates user profiles from different web applications to allow access to information in different user profiles in a unified way. We also present a UI that allows users to keep track of personal information stored in different applications. This helps users to control their personal data and thus it helps to prevent unintended data sharing. The paper is structured as follows: We first give an overview of related work in the field of user model aggregation and sharing. We then describe in detail our semantic approach to aggregate user models and how to access this information in a private and secure way. Finally, we show a system that uses the aggregated information to give users an overview about the personal information stored in different applications.

\section{State of the Art}

User information is typically stored in proprietary formats defined by each application. Thus, to give users a holistic view on their data, we need mechanisms to aggregate different user profiles. These aggregated user profiles have to be presented in a unified way to have an inter-application understanding of the stored information [2]. Such aggregated user profiles are also the basis for personalization of applications and recommendations [3]. In the research fields of user modeling and user model aggregation, different approaches have been proposed to address the problem of user model heterogeneity and aggregation. These approaches can be categorized into two types [4]:

- Standardization of user models: A common and shared user model standard for all applications.

- User model mediation: A set of techniques to transform or convert data from one user model to another format. This is a practical approach to solve the problem of heterogeneity and allows the aggregation of different models.

The standardization approach has a long research history starting with early works of simple user modeling shells [5] to more sophisticated user modeling servers [6,7]. Recent works propose a common user ontology, like GUMO [8], that creates a common syntax and understanding between all applications [9]. With the ontology as a shared data format, sharing of information is not a problem. But, this method is hardly possible in a commercial environment as most applications have application dependent user profiles. These profiles are adapted to the special requirements of each application and are firmly rooted within each system architecture. To change the structure of the user profile is always related with big changes to the system architecture, which means extra costs for the application providers.

The mediation approach [10] is a more practical approach. It aims to build a integrated user model suitable for a specific goal, e.g. recommendation of music. This integrated user model is based on data collected from different applications and aggregated by specialized software components. These software components transform data from one representation into a target representation. This approach solves the heterogeneity problem by having specialized software components for each 
transformation. The shortcoming here is that for each data field that should be transformed a separate component has to be developed which can lead to immense computational efforts.

\section{Semantic User-Centric Data Management}

In our approach, we focus on a hybrid strategy utilizing ideas from both types. To aggregate information, we developed an ontology, presented in section 3.1, as the basis for our system. For the sharing, we build a privacy-preserving framework, presented in section 3.3 and 3.4, which manages the data using different mediators and shares information between applications only with user consent. Figure 1 shows three user profiles with three attributes each, containing personal information and interest information, which should be aggregated utilizing our approach.
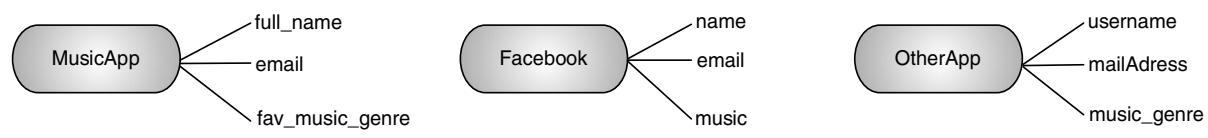

Fig. 1. Three different user profiles containing personal information and music interests

\subsection{The Aggregation Ontology}

We present a generic ontology, which allows us to define a degree of similarity between information and to determine the information source (the application that provided the data). Figure 2 visualizes our ontology. The ontology gives us the possibility to define a model, which describes how information in different profiles is related and how data can be aggregated. This aggregation model (AM) with descriptions about coherences between the user profile data is the basis for our system and for the in section 4 presented approach to show users their personal information stored in different applications.

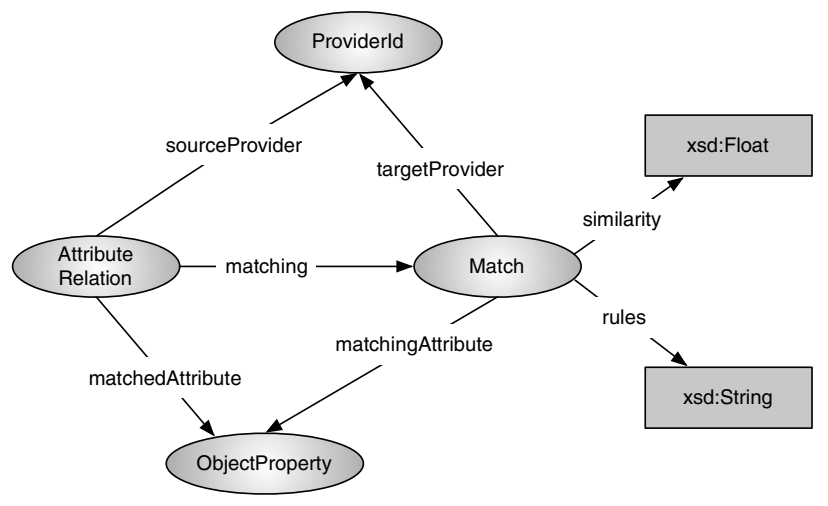

Fig. 2. Ontology for Profile Aggregation 
An important extension to existing ontological approaches, e.g. [11], is the entity Match, which allows adding extra knowledge to the model. Extra knowledge can be a similarity measure of related data in two different user profiles or instructions. Instructions can be a set of predefined rules [12] describing how to aggregate information. Such extra information is needed for the aggregation of user profiles, as it is an important indication for mediators how to handle the data. Table 1 gives a detailed description all entities and relations of the ontology.

Table 1. Entities and relations of the ontology

\begin{tabular}{|l|l|}
\hline Entity/Relation & Description \\
\hline ProviderId & The description of an UM provider with name and id. E.g. Facebook. \\
\hline AttributeRelation & $\begin{array}{l}\text { Defines a relation between the requested attribute (matchedAttribute) } \\
\text { of a provider (sourceProvider) with two or more attributes. } \\
\text { Connected attributes can be from different providers or only from } \\
\text { one provider. }\end{array}$ \\
\hline Match & $\begin{array}{l}\text { Encapsulates different attribute relations with extra information like } \\
\text { similarity, or rules how to aggregate data. }\end{array}$ \\
\hline owl:ObjectProperty & Defines the relations between instances of two classes. \\
\hline sourceProvider & The application identifier of the application hosting the UM. \\
\hline targetProvider & UM provider of the attribute similar to an requested attribute. \\
\hline matching & Relation between an AttributeRelation Entity and a Match Entity. \\
\hline similarity/rules & $\begin{array}{l}\text { The similarity attribute defines the degree of similarity between two } \\
\text { attributes. The rules attribute defines aggregation rules for the } \\
\text { profiles }\end{array}$ \\
\hline matchingAttribut & $\begin{array}{l}\text { Defines the special application attribute that corresponds to the } \\
\text { aggregation model attribute. }\end{array}$ \\
\hline matchedAttribut & $\begin{array}{l}\text { Defines the attribute that can be requested by other applications to } \\
\text { get similar attributes from different applications. }\end{array}$ \\
\hline
\end{tabular}

\subsection{User Profile Aggregation with the Ontology}

Based on this ontology, we can define a concrete model that allows us to aggregate user profiles from different applications and access the information in a unified way. To outline the approach, we exemplarily connect the three user profiles shown in Figure 1 (MusicApp, Facebook, OtherApp) containing personal (name, mail) and interest (music) information.

The actual definition of the model is a straightforward process. First, one has to analyze the given structure of the different user profiles that should be connected. The goal is to find similar attributes in different profiles that contain similar data. For attributes where the contained information is only partly related, a similarity measure has to be defined. The similarity measure is a substantial information for the data management and visualization process as it is an important indicator for the system how to handle the data. Such a similarity definition can be done manually, semiautomatically or automatically [13]. The aggregation of the profiles can be automatedo some extent [14]. In this scenario, we perform the aggregation manually. We have two information blocks, personal information and music interests, that can be aggregated. To aggregate the music information, we define a new AttributeRelation 
called 'music favorite genres' in our aggregation model (AM). We define matchedAttribute (AM\#music favorite genres) and sourceProvider (AM\#AM ID) entries accordingly, which are needed to access the model and retrieve information.

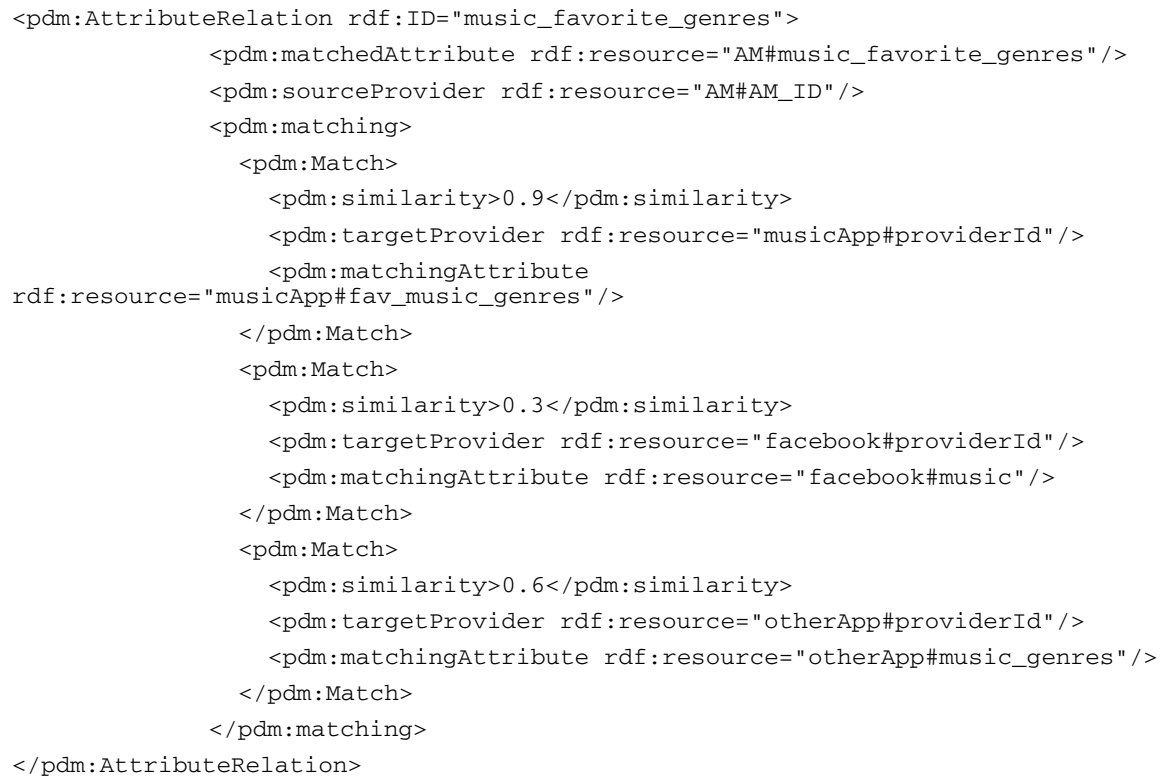

Fig. 3. Aggregation of music interests from 3 different profiles using the ontology

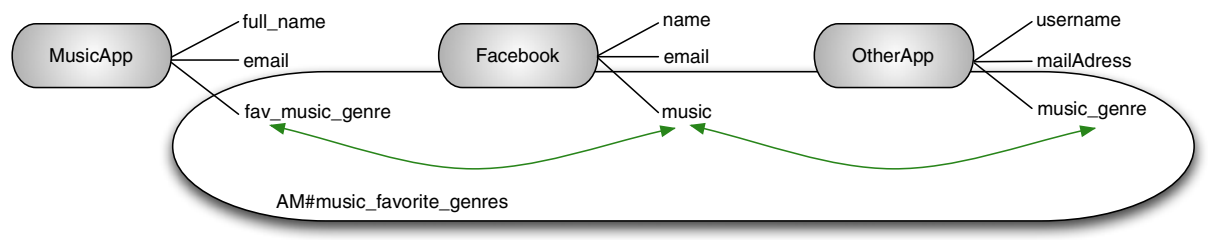

Fig. 4. The different music interests in the user profiles aggregated using the ontology

Fig. 3 shows the resulting model that describes the relations (Fig. 4) of the music interest attributes between the profiles. The aggregation of the personal information attributes follows this process. Once the model is defined, it is integrated into a framework that offers a web-service API to access the information.

\subsection{Privacy Aware Data Sharing}

The requirements for a privacy-aware approach sharing information safely across applications are to make sure that no personal user information is shared unintended. To fulfill these requirements, we utilize OpenID ${ }^{4}$. OpenID offers an interface to give permissions to third-party applications to use data and to actually share it. We have

\footnotetext{
${ }^{4}$ http://openid.net/
} 
chosen OpenID because it is a well established technology supported by companies like Google and Microsoft.

\subsection{Access to the Aggregated Data}

The actual data access is handled by the API which offers methods to request information on behalf of the user. Such a request can come from the user, who wants to access personal data or other systems that want to use the data for personalization or adaptation purposes. All access to the data must be confirmed by the user using the OpenId interface. If a system asks for information about 'AM\#music favorite genres', and the user approves the request, the system gets the information stored in the musicApp attribute 'musicApp\#fav music genres', the Facebook attribute 'facebook\#music' and the OtherApp data from 'OtherApp\#music genres' as these are related to the 'AM\#music favorite genres' field in the aggregation model.

\section{My Personal User Interface}

"My Personal User Interface" is a system that uses the presented ontology and framework, to visualize personal user information distributed over different applications. "My Personal User Interface" has the goal to assist users to

- keep track of applications they have,

- stay in control over their personal data,

- control the information flow of personal data.

To support people to have an overview over their applications and data the main UI is split into a top and bottom view, see Figure 5. The top view is a cover-flow element showing the different applications of a user. The cover-flow allows selecting an application and getting an overview of the personal information stored in it. This personal information is presented in the bottom view of the UI. For example, Figure 5 shows personal contact information stored in Facebook. We adopted the information card metaphor [15] to visualize the different applications of the user and to visualize the stored personal information.

Figure 6 gives an example of the type of information and how it is visualized in our system. The user has different information cards visualizing information stored about her. The user can see her last actions in an application, as an example of implicit information visualized by our system.

We also designed an UI to support users in privacy-aware use-cases, see Figure 7, which allows users to control what kind of data is distributed. If a third-party application requests data of the user, the user is asked to give permission for that application to use the data. Therefore, the UI presents data that will be sent to the user. The process of sharing information is two-folded. After a third-party application requests data, e.g. about the user's musical taste, the system selects all information previously aggregated using the ontology. So, for a music taste request, data from Facebook or the previously described 'Profile Analysis'-profile would be selected. The second step is the validation through the user. Therefore, the selected information is presented to the user using the same card metaphor but only showing the information to be sent to the 


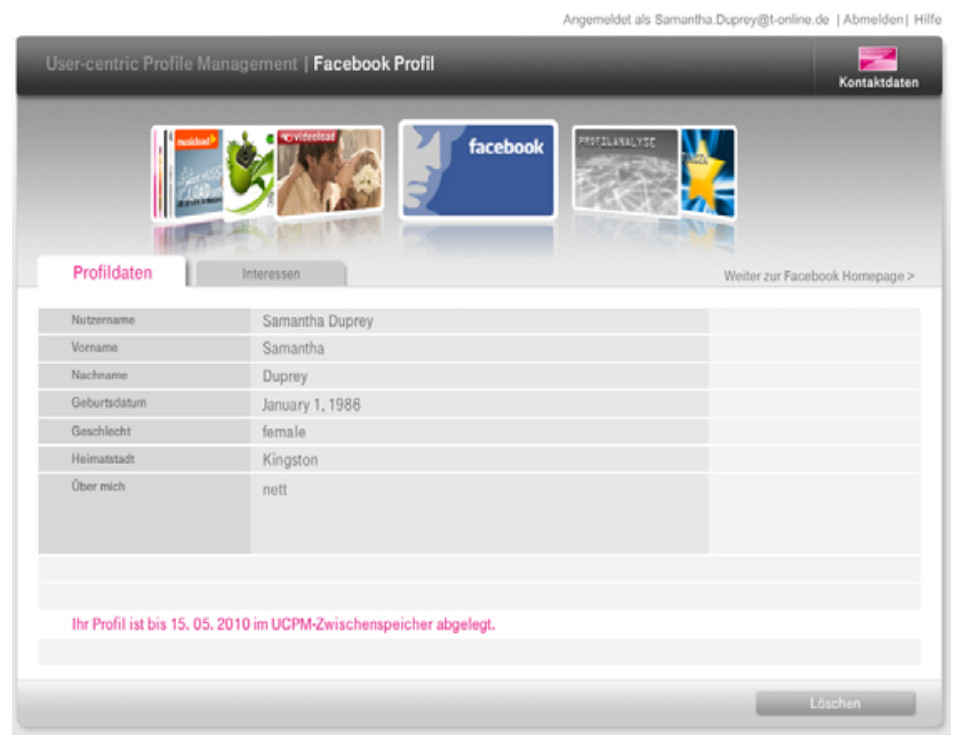

Fig. 5. Main UI showing different applications and personal information

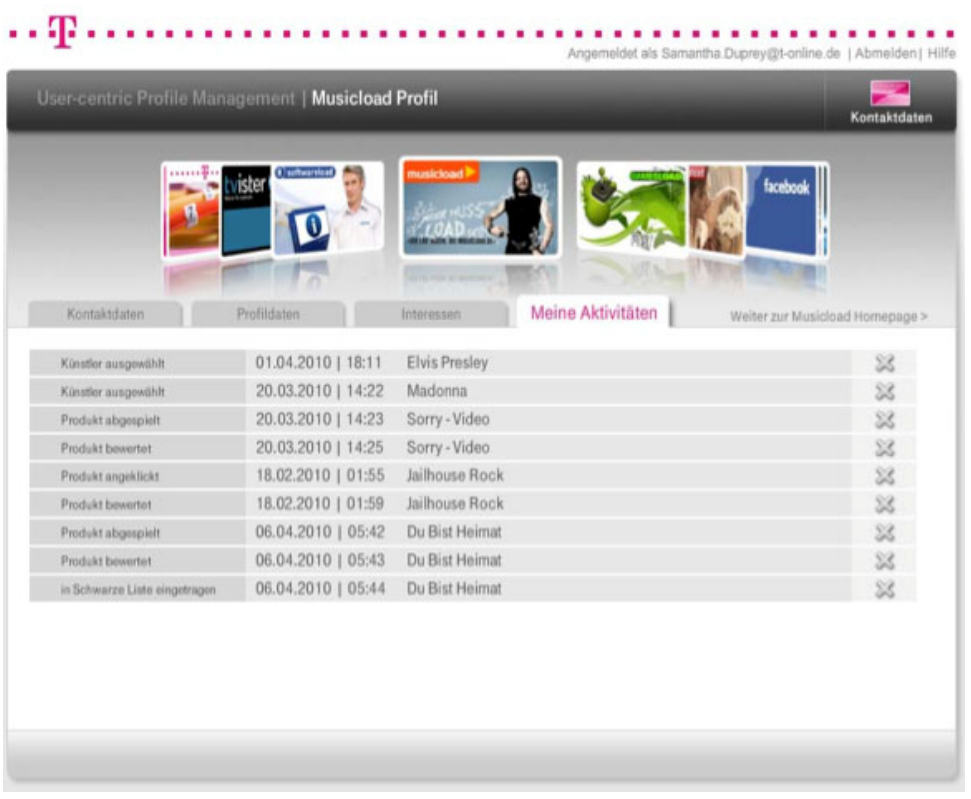

Fig. 6. Visualization of past behavior

third-party application. The user can navigate through the different cards, see which information will be sent and decide to accept or deny the request. This makes it possible to easily see and control what data is shared. 


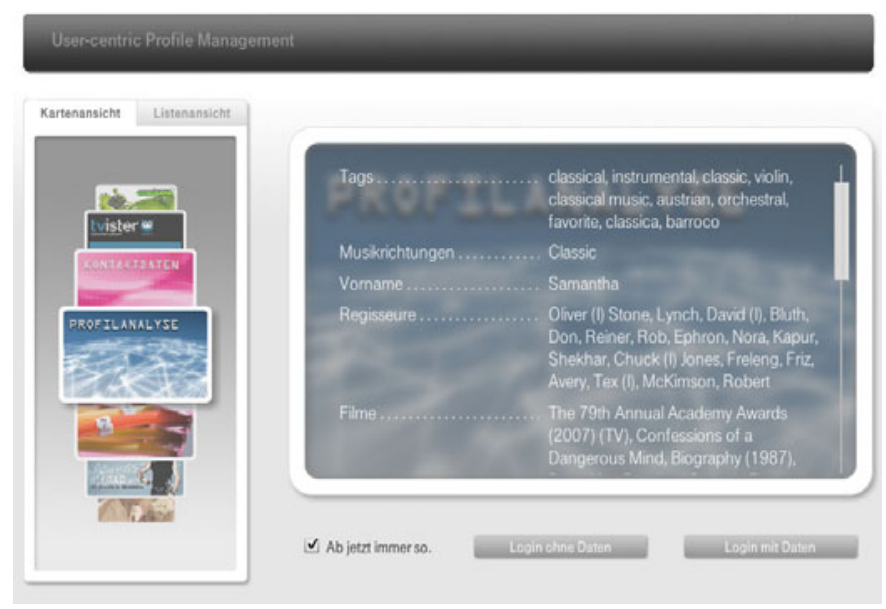

Fig. 7. Our OpenId interface. Buttons at the bottom allow users to accept or deny a data request.

\section{Conclusion and Outlook}

In this paper we introduced a solution to aggregate, access and manage personal information in a secure way. We presented a new ontology that supports the aggregation of distributed user profiles and a framework that utilizes the ontology and allows secure data sharing by using OpenId. On top of this framework, we created My Personal User Interface, a system that allows users to fully profit from the semantic framework and to keep an overlook over their personal data. In future steps, we want to to use the aggregated user information to further enrich the knowledge about the user and add new information retrieved from the Semantic Web. This information can be used to improve personalization and adaption and help users to profit more from such a personalized system. Of course, the user stays in control and can decide to discard this automatically added information.

\section{References}

1. Abel, F., Henze, N., Herder, E., Krause, D.: Interweaving Public User Profiles on the Web. In: De Bra, P., Kobsa, A., Chin, D.N. (eds.) UMAP 2010. LNCS, vol. 6075, pp. 16-27. Springer, Heidelberg (2010)

2. Plumbaum, T.: Semantically-Enhanced Ubiquitous User Modeling. In: De Bra, P., Kobsa, A., Chin, D.N. (eds.) UMAP 2010. LNCS, vol. 6075, pp. 407-410. Springer, Heidelberg (2010)

3. Plumbaum, T., Lommatzsch, A., Rudnitzki, S., Luca, E.W.D., Dwiger, H., Albayrak, S.: Adaptive music news recommendations based on large semantic datasets. In: 1st Workshop On Music Recommendation And Discovery (WOMRAD), ACM RecSys, 2010, Barcelona, Spain (2010) 
4. Kuflik, T.: Semantically-enhanced user models mediation: Research agenda. In: 5th International Workshop on Ubiquitous User Modeling (UbiqUM 2008) (January 2008)

5. Finin, T.W.: Gums: A general user modeling shell. In: Kobsa, A., Wahlster, W. (eds.) User Models in Dialog Systems, pp. 411-430. Springer, Heidelberg (1989)

6. Kay, J., Kummerfeld, B., Lauder, P.: Personis: A Server for User Models. In: De Bra, P., Brusilovsky, P., Conejo, R. (eds.) AH 2002. LNCS, vol. 2347, pp. 203-212. Springer, Heidelberg (2002)

7. Fink, J.: User Modeling Servers Requirements, Design, and Evaluation. PhD thesis, Universitt Duisburg-Essen (2003)

8. Heckmann, D., Schwarzkopf, E., Mori, J., Dengler, D., Krner, A.: The user model and context ontology gumo revisited for future web 2.0 extensions. In: Bouquet, P., Euzenat, J., Ghidini, C., McGuinness, D.L., Serafini, L., Shvaiko, P., Wache, H. (eds.) Proceedings of the International Workshop on Contexts and Ontologies: Representation and Reasoning (CO:RR). CEUR Workshop Proceedings, CEUR-WS.org, vol. 298 (2007)

9. Gruber, T.R.: Toward principles for the design of ontologies used for knowledge sharing. Int. J. Hum.-Comput. Stud. 43(5-6), 907-928 (1995)

10. Berkovsky, S., Kuflik, T., Ricci, F.: Mediation of user models for enhanced personalization in recommender systems. User Modeling and User-Adapted Interaction 18(3), 245-286 (2008)

11. van der Sluijs, K., Houben, G.J.: A generic component for exchanging user models between web-based systems. Int. J. Continuing Education and Liflong Learning 16(1/2), 64-76 (2006)

12. Leonardi, E., Abel, F., Heckmann, D., Herder, E., Hidders, J., Houben, G.J.: A Flexible Rule-Based Method for Interlinking, Integrating, and Enriching User Data. In: Benatallah, B., Casati, F., Kappel, G., Rossi, G. (eds.) ICWE 2010. LNCS, vol. 6189, pp. 322-336. Springer, Heidelberg (2010)

13. Banerjee, S., Pedersen, T.: Extended gloss overlaps as a measure of semantic relatedness. In: Proceedings of the 18th International Joint Conference on Artificial Intelligence, pp. 805-810. Morgan Kaufmann Publishers Inc., San Francisco (2003)

14. Noy, N.F.: Semantic integration: a survey of ontology-based approaches. SIGMOD Rec. 33, 65-70 (2004)

15. Ballinger, K., Bill Barnes, G.S., Causey, J.: Patterns for supporting information cards at web sites: Personal cards for sign-up and sign-in. Technical report, Microsoft (2007) 